\title{
PROPERTIES OF SOME SUBSEQUENCES OF THE WALSH-KACZMARZ-DIRICHLET KERNELS
}

\author{
NACima Memić
}

Abstract. We study some properties of a family of subsequences of the Walsh-Kaczmarz-Dirichlet kernels. We prove properties related to the $L^{1}$ norm of the weighted maximal function and to the Fejér means of partial sums of Fourier series obtained by convolution with integrable functions.

Mathematics subject classification (2010): 42C10.

Keywords and phrases: Walsh-kaczmarz system, partial sums of Fourier series, weakly bounded operator.

\section{REFERENCES}

[1] E. S. Belins Ky, Summability of Fourier series with the method of lacunary arithmetical means at the Lebesgue points, Proc. Am. Math. Soc. 125 (1997), no. 12, 3689-3693.

[2] D. L. Burkholder, Distribution function inequalities for martingales, Ann. Probability 1(1973), $19-42$.

[3] N. J. Fine, Cesàro summability of Walsh-Fourier series, Proc. Nat. Acad. Sci. U.S.A. 41 (1955), 558-591.

[4] G. GÁT, Almost everywhere convergence of Fejér and logarithmic means of subsequnces of partial sums of the Walsh-Fourier series of integrable functions, J. of Approx. Theory 162 (2010), 687-708.

[5] G. GÁt, On the Calderón-Zygmund decomposition lemma on the Walsh-Paley group, Acta Math. Acad. Paedagog. Nyházi. (N.S.) 14 (1998), 25-30.

[6] G. GÁT, On the $L^{1}$ norm of the weighted maximal function of the Walsh-Kaczmarz-Dirichlet kernels, Acta Acad. Paedagog. Agriensis, Sect. Mat. (N.S.) 30 (2003), 55-65.

[7] U. Goginava, K. NAGY, Maximal operators of Fejér means of Walsh-Kaczmarz Fourier series, J. Funct. Space. Appl Vol 8, 2 (2010), 181-200.

[8] F. Schipp, W. R. WAde, P. Simon, J. PÁL, Walsh series: an introduction to dyadic harmonic analysis, Adam Hilger, Bristol and New York, 1990.

[9] W. F. Stout, Almost sure convergence, Academic Press, New York, 1974.

[10] W. S. Young, Mean convergence of generalized Walsh-Fourier series, Trans. Amer. Math. Soc, 218 (1976), 311-320.

[11] Z. ZalCwasser, Sur la sommabilité des séries de Fourier, Stud. Math. 6 (1936), 82-88. 